\title{
Index Finger Movement Imitation by Human Neonates: Motivation, Learning, and Left-Hand Preference
}

\author{
EMESE NAGY, HAJNALKA COMPAGNE, HAJNALKA ORVOS, ATTILA PAL, PETER MOLNAR, \\ IMRE JANSZKY, KATHERINE A. LOVELAND, AND GYORGY BARDOS
}

\begin{abstract}
Department of Psychology [E.N.], University of Dundee, Nethergate, DD14HN, Dundee, Scotland, United Kingdom; Department of Physiology and Neurobiology [H.C., G.B.], Eötvös Loránd University, Budapest, H-1117 Hungary; Department of Obstetrics and Gynecology [H.O., A.P.], Albert Szent-Gyorgyi Medical University, 6723, Szeged, Hungary; Department of Behavioral Sciences [P.M.], University of Debrecen, Debrecen, Hungary; Department of Behavioral Sciences [I.J.], Semmelweis University, 1088, Budapest, Hungary; and Center for Human Development Research [K.A.L.], Department of Psychiatry and Behavioral Sciences, University of Texas Medical School at Houston, Houston, TX 77030
\end{abstract}

\section{ABSTRACT}

Imitation of a fine motor movement, index finger protrusion, was examined in 39 neonates using an ethologically based objective coding system. Results confirmed that imitation of finger movements exists, and infants demonstrated "learning" as imitation developed through an incomplete imitation stage. Neonatal imitation was more frequently left-handed, an early sign of laterality in motivation to be investigated further. The existence of index finger imitation in human neonates indicates that volitional control of individuated finger movements develops much earlier than previously thought. The differential increase of index finger protrusion movements during the imitation periods suggests that this behavior is not an automatic response triggered by general arousal but instead is a true indicator of purposeful neonatal imitation. (Pediatr Res 58: 749-753, 2005)
The cognitive developmental approach in the first half of the 20th century (1) assumed that imitation of observed actions occurs at the earliest from 10-12 mo of age, based on learning and experience. Since the 1970s, a series of studies (2-9) has confirmed that infants even as young as a few hours old can imitate proprioceptively guided behaviors. Despite these findings, neonatal imitation remained a controversial "fuzzy phenomenon" (10). Several studies failed to replicate the phenomenon (11-14), and it was even regarded as artefact by some researchers (15). The confusion about neonatal imitation in part originates from the fact that most movements of neonates were regarded as unintentional until Van der Meer et al. (16) showed that newborn infants can purposefully control their arm resistance against small forces. Recent research showed that most neonatal behaviors, including neonatal reflexes, such as rooting, sucking, and imitation, are flexible and goal-directed behaviors (17). Nagy and Molnar $(18,19)$ reported that neonates not only are able to imitate tongue protrusion gestures but

Received September 22, 2004; accepted February 11, 2005.

Correspondence: Emese Nagy, M.D., Ph.D., Department of Psychology, University of Dundee, Nethergate, Dundee, DD1 4HN, Scotland, UK; e-mail: E.Nagy@dundee.ac.uk.

This study was supported by grant OTKA F023194 from the Hungarian National Research Foundation.

DOI: 10.1203/01.PDR.0000180570.28111.D9 also started to initiate the previously imitated gestures and to engage in long-lasting imitation/initiation "conversation" with the experimenter. Imitations and initiations were coupled with opposite heart rate changes during these dialogues and may indicate a prospective control of a new movement (heart rate increase during imitations) and an attention to an expected reply (heart rate decrease during initiations).

Imitation is still lacking an ethologic, and neurophysiologic description in neonates. Why newborn infants imitate and how they can do it are still unanswered questions, although various models have been proposed. Imitation has been described as an unconditioned reflex, a circular reaction (20) or a secondary circular reaction (1), and as an innate releasing mechanism (21). It has been explained as a product of associative learning (22) or instrumental learning (23). Recent explanations include the theory of intermodal mapping $(3,24)$ and the mirror neuron system model $(25,26)$. Although these models successfully explain several aspects of neonatal imitation, none of them is able to describe how imitation happens, and they also failed to explain why infants start to imitate or what motivates their first imitations.

The majority of studies with neonates used the tongue protrusion gesture $(3,6,7,9,27,28)$. Fewer studies involved other facial movements, such as lip and mouth movements 
$(3,27)$, imitation of emotional expressions (4), and other gestures such as head turning (28) or vocalization (9). Very few studies with young infants used leg and hand movements $(2,29)$. In the history of neonatal imitation research, imitation of body movements that infants cannot observe on themselves (e.g. tongue protrusion) was theoretically important, because the Piagetian theory hypothesized its emergence months later than that of movements that infants can directly observe (e.g. hand movements). However, focusing on tongue protrusion imitation has various limitations. The baseline frequency of this movement is very high, and spontaneous and imitative movements cannot be easily distinguished (27). In addition, in these experiments, the face, the primary interface for social communication, is "used" as a tool during tongue protrusion imitation, further increasing the difficulty of exploring the interpersonal functions of neonatal imitation.

Using fine motor hand movements, such as independent finger movements, could help to overcome these limitations. Finger movements as imitative gestures have not yet been studied and described in human neonates in part because many investigators have focused on challenging Piaget's model of imitation and also because it has been assumed that independent finger movements develop much later, between 6 and 12 mo after birth (30). Independent finger imitation, if exists in newborn infants, could shed new light on the nature of neonatal imitation. First, as a low-frequency baseline movement, it could be objectively described and easily coded and therefore could become a convincing proof for the existence of neonatal imitation. Second, because independent finger movement imitation is a relatively difficult fine motor movement, studying it would enable us to investigate the learning component of neonatal imitation. Third, the laterality of the imitation (whether it is predominantly left or right, or specular, i.e. mirror; or anatomic, e.g. left finger imitation as a response to a left finger movement of the model) could further help the exploration of the neural bases of neonatal imitation. Finally, because the movement is separate from the face, the interpersonal functions of this kind of imitation could also be more readily studied.

This means that while manual imitations are performed, the face of the experimenter and the newborn are not the "tool" of the imitation any more. Infants have the choice to turn toward the experimenter or away or look at or not look at the experimenter. Infants do not have to engage in face-to-face contact during hand movement imitations, whereas they must do soeven if briefly-to perform facial imitations. If imitation is interactive by its nature, then infants' seeking additional eye contact and turning toward the experimenter may support the notion that imitation is motivated behavior serving interaction and not merely a reflexive phenomenon.

In this study, the presence and the nature of independent finger movement imitation were examined in human neonates. We randomly modeled left- and right-handed index finger extension movements to newborns. The index finger extension gesture was chosen for several reasons. This gesture is similar to those used in previous studies that described the mirror neuron system model of imitation $(31,32)$. Imitative responses to this particular gesture can vary in laterality (left, right, or mixed) and in the relationship of the imitative movement to the relative position of the infant and the experimenter (specular, anatomic, or random imitation). Gestures may also vary in accuracy, that is, how similar they are to the modeled gesture. Therefore, the predominant pattern of neonatal imitation can offer an insight into its mechanism and nature. Finally, index finger extension movement is a fine motor movement with a relatively low spontaneous occurrence, which allows for an ethologically based, frame-by-frame, quantitative coding procedure to confirm the specificity of the neonatal imitation. This coding procedure used detailed descriptions that are sensitive to complex variations of movements, seeking patterns without restrictive a priori definition of "responses." Therefore, this ethologic based coding system is able to describe movements without hypothesizing their functional or emotional significance and meaning.

\section{METHOD}

Subjects. With the informed consent of the mothers, 43 healthy newborn infants ( 23 boys and 20 girls) were examined in the first 3-96 h of their lives. The study was approved by the Institutional Review Board of the Albert Szent-Gyorgyi Medical University. Data from four infants were excluded from the study. Two infants were excluded by the coders because they were sleeping, and two other infants were excluded because of low interrater reliability on coding. Thirty-nine infants were included in the final analysis. Infants were born at an average of 38.57 gestational weeks (SD $\pm 0.85 ; 36-41$ wk); 26 were born through vaginal delivery, and 13 were delivered by cesarean section.

Procedure. The examination room, which was a separate but integral part of the neonatal ward, had constant illumination and ambient temperature $\left(28^{\circ} \mathrm{C}\right)$, and the conditions and the environment of the room were the same for every newborn. Newborns were examined 30-90 min after feeding, which proved to be the optimal time for examining them in an awake but quiet state.

Infants first were placed on their back on an examining table with their head turned toward the left side, facing a Panasonic 240 type video camera and the experimenter. A mirror was placed on the right side of the infant so that the experimenter could be seen by the video camera. For assessing the effect of head and body position in imitation, all infants were turned to the right side for the second part of the study while the mirror stayed at the right side. The experimenter varied the side of the hand presented as a model. Experiments lasted for an average of $24.68 \mathrm{~min}(\mathrm{SD} \pm 2.29)$. The baseline period was an average of $4.42 \mathrm{~min}$ ( $\mathrm{SD} \pm 1.49 \mathrm{~min}$ ), after which the experimenter showed an index finger protrusion movement to the infant. In the response period, the experimenter waited for an average of $50 \mathrm{~s}$ and then administered the next gesture. An average of 25 imitation periods were initiated by the experimenter. The frequency and the duration of the hand movements, both of the experimenter and of the infant, were coded from time-stamped video records by a naive coder.

Coding. Video records were coded by a naive coder who was unaware of the main purpose of the experiment. All hand and finger movements on the video records were coded regardless of whether they were during the imitation or the baseline period and regardless of whether the movements were imitative or spontaneous. As both direct and mirror views of the infants' hands were visible to the coders, all finger movements of the infants could be observer clearly regardless of the infant's posture. The purpose of this coding was to confirm the existence of imitation with accurate descriptions of the hand movements. Beginning and end times of every hand movement of the infant and the experimenter were coded. Infants' finger movements were additionally coded using a three-level coding system $(1=$ infant raises hand; $2=$ incomplete index finger extension movement or index finger extension movement accompanied by extensions of one or two other fingers; $3=$ complete index finger extension movement with only the index finger raised). The laterality of the experimenter's movements and the infants' incomplete and complete index finger movements were also coded. One hundred percent of the data were recoded for reliability using frame-by-frame coding, and an $87 \%$ interrater reliability was attained.

Recorded variables. Hand movements of the infants (code 1) and finger movements of the infant (code 2 for incomplete and code 3 for complete finger movements) and laterality of the finger movements in neonates (left or right) and for the experimenter were coded. The average response time in seconds for 
the first, second, and third imitations were also calculated. Response time was the time between the end of the experimenter's gesture and the beginning of the infants' responses. Mean heart rate was measured in beat $/ \mathrm{min}$. Data were examined using SPSS 10.0 Statistical software and a $p<0.05$ level of significance was accepted throughout.

\section{RESULTS}

Infants made more hand and finger movements in the imitation period [repeated measures $\operatorname{ANOVA}, \mathrm{F}(1,38)=35.08, p$ $<0.001]$ and more nonspecific hand movements than specific index finger extension gestures during both the imitation and the baseline periods $[\mathrm{F}(2,37)=52.26, p<0.001$; Fig. 1]. However, as evidence of the intention to imitate and learning, infants increased the frequency of finger movements (incomplete and complete finger extensions) during the imitation period $[\mathrm{F}(2,37)=3.39, p<0.05]$ compared with the baseline period. The proportion of all coded movements that were finger movements (incomplete and complete finger extensions) compared with the nonspecific hand movements, significantly increased in the imitation period (from 21.11 to $28.97 \%$ of all movements). The average response time was $\mathrm{M}=25.23 \mathrm{~s}$ (SD $=45.93)$ at the first, $\mathrm{M}=8.21 \mathrm{~s}(\mathrm{SD}=16.01)$ at the second, $\mathrm{M}=21.64 \mathrm{~s}(\mathrm{SD}=48.08)$ at the third, $\mathrm{M}=4.93 \mathrm{~s}(\mathrm{SD}=$ $6.07)$ at the fourth, and $\mathrm{M}=12.61 \mathrm{~s}(\mathrm{SD}=19.82)$ at the fifth imitation. Overall, response time decreased through the subsequent imitations [repeated ANOVA, F(4, 35) $=6.03, p<$ $0.01]$.

Movements in the imitation period showed gradual improvement, that is, increasing refinement of the movement toward the modeled gesture. Only eight $(20.51 \%)$ of the 39 infants imitated perfect index finger extension during the first imitation period. Infants performed their first incomplete index finger protrusion movement at about the fourth imitation period $(\mathrm{M}=$ $3.92, \mathrm{SD} \pm 1.32$ ) but produced their first complete index finger movement significantly later, at approximately the sixth or seventh imitation period $(\mathrm{M}=6.27, \mathrm{SD} \pm 2.14, \mathrm{t}=-2.18, p$ $=0.036)$.

Infants' index finger movements were predominantly left sided during the imitation period but not during the baseline period [repeated ANOVA, side*time interaction; $\mathrm{F}(1,38)=$ $6.31, p=0.016]$. This left-handed dominance of finger movements during the imitation period was not related to the infant's

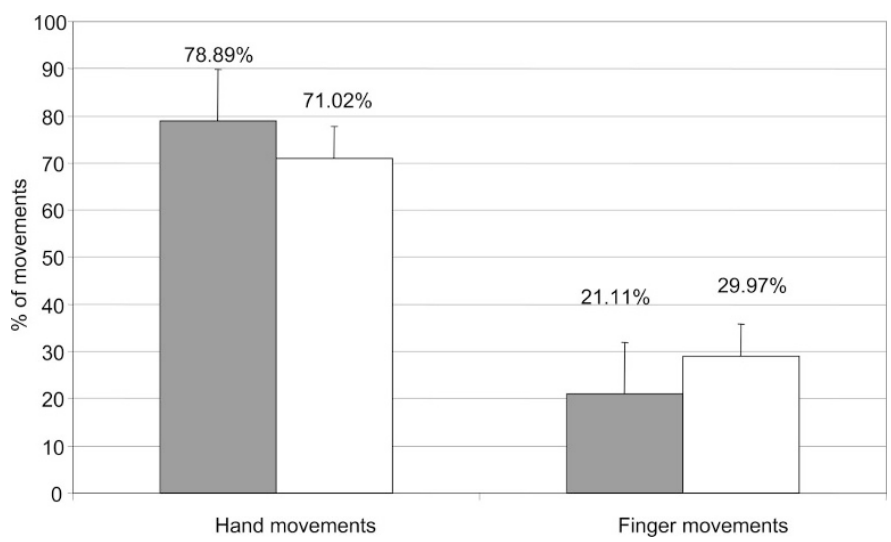

Figure 1. The frequency of hand and finger movements before and during imitation. 䍔, Before imitation; $\square$, during imitation. position [whether infants were turned to their left or right side; side $*$ position interaction, $\mathrm{F}(1,38)=1.49$; NS; Fig. 2). Infants made left-handed imitative index finger movements without mirroring the experimenter's handedness [experimenter's hand movement laterality * infant's hand movement laterality interaction, $\mathrm{F}(1,38)=1.90$; NS].

In summary, these behavioral data showed that the frequency of index finger protrusion movements specifically increased during the imitation period and that infants imitated the gesture with decreasing response time and increasing accuracy. It is interesting that infants' finger movements were predominantly left-handed. The differential increase of index finger protrusion movements during the imitation period suggests that this behavior pattern is not an automatic response triggered by general arousal but instead is a true indicator of neonatal imitation.

\section{DISCUSSION}

Findings of this study indicate that newborns are able to imitate index finger extension movements. The specific increase of incomplete and complete index finger movements as opposed to general hand movements suggests that imitation cannot be explained solely by increased motor activity caused by general arousal. Infants started to imitate relatively quickly, and imitations progressed through an incomplete imitation stage before the attainment of accurate imitation. With subsequent imitations, the accuracy gradually increased from general hand movements to incomplete finger movements toward the complete finger movement imitation. The finding of a left-handed preference indicates that, at some level, a lateralized system motivates neonatal imitation.

The results also showed that independent finger movements exist immediately after birth, long before the second half of the first year, as previously thought. The corticospinal projections that are necessary for these movements are known to be established as early as the 24th postconceptional week, and the functional monosynaptic projections and the extensive innervations of the spinal neurons before birth provide the capacity for independent finger movements (33). These movements have not yet been adequately described.

Our results are interesting not only because we confirm the presence of voluntary imitative finger movements in newborn

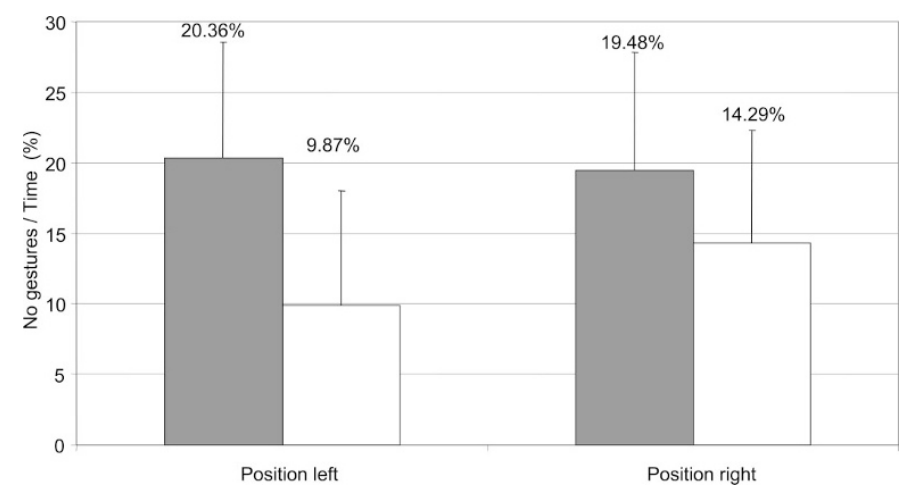

Figure 2. Lack of relationship between left-handed imitation position. Left hand; $\square$, right hand. 
infants but also because we found evidence that imitative responses could be produced more rapidly than expected in neonates. Newborn infants are thought to be very slow (10) in their social responses. Heimann found that tongue protrusion imitation started only $\sim 1$ min after modeling, whereas in our study, finger movement imitations appeared in an average of a half minute after the model. Central conduction time of the impulse for finger movements in newborns is indeed slower (three times slower) than those of adults, but their movements are not necessarily slower (33).

The more rapid response times observed in our study could result from differences in experimental procedures used. In the pioneer studies of Meltzoff and Moore (3) and Heimann (28), the experimenter's continuous modeling was relatively insensitive to the infant's condition, attention, and response. Our study was more interactive and responsive to the infants' condition and attention, and this experimental design is closer to the natural, communicative function, of early imitation (34-36). Thus, infants may have been more responsive, motivated, and faster because imitation occurred in an interpersonal interactive setting. In naturally occurring interaction, this speed could enable infants to respond fast enough to be competent communicators in an address-and-reply cycle.

Results from this study showed that with succeeding imitations, infants became faster and more accurate. Previous studies did not continue the imitation experiments through a cycle of imitations, or those that did have not analyzed the change of imitative response patterns over time. Also, most studies used a tongue protrusion gesture, which does not substantially change from response to response and is an easy motor act for infants. Abravanel and Sigafoos (8) reported that in their study, imitations were always partial and incomplete. If our study had stopped at the first imitations, then the results would probably support Abravanel and Sigafoos's argument; however, infants changed their behavior over subsequent imitations, performing more and more complete and accurate movements with decreasing response times. However, for methodologic clarity, in further studies, control conditions, such as opened and semiopened hand movement models- besides the finger extension movements - should be introduced for a more accurate description of the phenomenon.

Demonstrations of functional activity in the cortex of neonates permit us to speculate about whether the mirror neuron system (37) or elements related to it contribute to the neurophysiologic bases of neonatal imitation. The mirror neuron system of adults has lateralized components, including Broca's area of the left hemisphere and bilateral dorsal and ventral premotor areas, the right superior temporal gyrus, and supplementary motor area, these being described as elements of a common circuit for imitation of various forms of movements (32). Neurophysiologic studies confirm that the cortex of the newborn infant is functionally active, although immature. The mismatch negativity event-related potential over the temporal areas occurs at $292 \mathrm{~ms}$ in newborns (38), slower than the $80-160 \mathrm{~ms}$ in adults (39). The temporal lobe, however, does generate this potential in newborns (40), and most ERP components can be elicited soon after birth, even if with longer latency (41). This means that cortical generators of the ERP peaks are functioning at birth, and the functional activity of various cortical areas can contribute to the emergence neonatal imitation and may indirectly support a mirror neuron hypothesis.

The mirror neuron system in humans could be a possible evolutionary basis of evolving language (42). It may be plausible that language originates from the mirror neuron system not only in the evolutionary sense but also ontogenetically. In an earlier study, we described a phenomenon that we called neonatal initiation or provocation $(19,20)$. In that study, using tongue protrusion gesture, we found that newborns not only imitated the gesture but after a while started to initiate the previously imitated gestures, waiting for the experimenter to respond $(19,20)$. These imitation/initiation cycles developed into an overlapping communication sequence, the laboratory model of the first "dialogue." This finding therefore suggests that early imitative dialogues in a natural setting help infants to master communicative turn-taking and to engage in long "conversations" long before language appears.

If the mirror neuron system subserves the evolutionary and possibly ontogenetic roots of communication development, if we regard neonatal imitations as the first "conversations," and if the cortical areas of newborn infants are functionally active, then it is plausible that the mirror neuron system contributes to early imitations. In this study, we found predominantly leftsided index finger imitations. Left-sided finger imitations could be explained by a left-sided mirror neuron system if we take into account the potential role of the ipsilateral motor pathway in the movement. The left ipsilateral motor pathway has a stronger influence on complex fine motor movements even in adults (43), and in young infants, the ipsilateral motor pathway is faster than the contralateral (30); therefore, it is a possible (if unlikely) explanation for the reported left-sided bias. The role of the right hemisphere is an additional potential explanation for left-handed imitative movements. The right hemisphere is dominant in emotional and face processing $(44,45)$, and emotional facial expressions in humans (46) and in chimpanzees (47) are also stronger on the left side. Thus, right hemisphere dominance for a hand gesture may suggest that neonatal imitation is not a simple isopraxism but a motivated expressive behavior.

Because of the limitations of the experiment, the possible lateralization of early imitative responses must be investigated further. For further studies, the use of the index finger imitation gesture, as described and verified in our study, could offer an excellent tool to explore the mechanism of neonatal imitation.

Acknowledgments. We thank the reviewers and Prof. Colwyn Trevarthen for help in writing and editing this article.

\section{REFERENCES}

1. Piaget J 1962 Play, Dreams and Imitation in Childhood. Norton, New York

2. Maratos O 1973 The origin and development of imitation in the first six months of life. Paper presented at the Annual Meeting of the British Psychological Society; April 1973; Liverpool

3. Meltzoff AN, Moore MK 1977 Imitation of facial and manual gestures by human neonates. Science 198:74-78

4. Field TM, Woodson R, Greenberg R, Cohen D 1982 Discrimination and imitation of facial expression by neonates. Science 218:179-181 
5. Fontaine R 1984 Imitative skills between birth and six months. Infant Behav Dev 7:323-333

6. Reissland B 1988 Neonatal imitation in the first hour of life: observation in rural Nepal. Dev Psychol 24:464-469

7. Heimann M, Schaller J 1985 Imitative reactions among 14-21 day old infants. Infant Mental Health J 6:31-39

8. Abravanel E, Sigafoos AD 1984 Exploring the presence of imitation during early infancy. Child Dev 55:381-392

9. Kugiumutzakis G 1999 Genesis and development of early infant mimesis to facial and vocal models. In: Nadel J, Butterwirth G (eds) Imitation in Infancy. Cambridge University Press, New York, pp 22-47

10. Heimann M 2001 Neonatal imitation-a fuzzy phenomenon? In: Lacerda F, von Hofsten C, Heimann M (eds) Emerging Cognitive Abilities in Early Infancy. Lawrence Erlbaum Associates, London, pp 231-246

11. Hayes LA, Watson JG 1981 Neonatal imitation: fact or artifact? Dev Psychol 177:660-665

12. Masters JC 1979 Interpreting "imitative" responses in early infancy. Science 205:215

13. McKenzie B, Over R Young infants fail to imitate manual and facial gestures. Infant Behav Dev 6:85-95, 1983

14. Anisfeld M, Turkewitz G, Rose SA, Rosenberg FR, Sheiber FJ, Couturier-Fagan DA, Ger JS, Sommer I 2001 No compelling evidence that newborns imitate oral gestures. Infancy 2:111-122

15. Anisfeld M 1979 Interpreting "imitative" responses in early infancy. Science 205:214-215

16. van der Meer AL, van der Weel FR, Lee DN 1995 The functional significance of arm movements in neonates. Science 267:693-695

17. von Hofsten C 2004 An action perspective on motor development. Trends Cogn Sci $8: 266-272$

18. Nagy E, Molnar P 2004 Homo imitans or homo provocans? The phenomenon of neonatal initiation. Infant Behav Dev 27:57-63

19. Nagy E, Molnar P 1994 Homo imitans or homo provocans? Int Psychophysiol 18:128

20. Baldwin JM 1902 Development and Evolution. Macmillan, New York

21. Jacobson SW 1979 Matching behavior in the young infant. Child Dev 50:425-30

22. Thorndike EL 1898 Animal intelligence. An experimental study of the associative processes in animals. Psychol Rev Mon 2:551-553

23. Miller NE, Dollard J 1941 Social learning and imitation. Yale University Press, New Haven

24. Melztoff AN 1988 The human infant as homo imitans. In: Social Learning Psychological and Biological Perspectives. Hillsdale, New Jersey, pp 318-340

25. Iacoboni M, Woods RP, Brass M, Bekkering H, Mazziotta JC, Rizzolatti G 1999 Cortical mechanisms of human imitation. Science 286:2526-2528

26. Wohlschlager A, Bekkering H 2002 Is human imitation based on a mirror-neurone system? Some behavioural evidence. Exp Brain Res 143:335-341

27. Meltzoff AN, Moore MK 1983 Newborn infants imitate adult facial gestures. Child Dev 54:702-709

28. Heimann M, Nelson KE, Schaller J 1989 Neonatal imitation of tongue protrusion and mouth opening: methodological aspects and evidence of early individual differences. Scand J Psychol 30:90-101
29. Meltzoff AN, Moore MK 1997 Explaining facial imitation: a theoretical model. Early Dev Parenting 6:179-192

30. Eyre JA, Miller S, Clowry GJ, Conway EA, Watts C 2000 Functional corticospinal projections are established prenatally in the human foetus permitting involvement in the development of spinal motor centres. Brain 123:51-64

31. Iacoboni M, Koski LM, Brass M, Bekkering H, Woods RP, Dubeau MC, Mazziotta JC, Rizzolatti G 2001 Reafferent copies of imitated actions in the right superior temporal cortex. Proc Natl Acad Sci USA 98:13995-13999

32. Leslie KR, Johnson-Frey SH, Grafton ST 2004 Functional imaging of face and hand imitation: towards a motor theory of empathy. Neuroimage 21:601-607

33. Schieber MH 1996 Individual finger movements. In: AM Wing, Haggard P, Flanagan JR (eds) Hand and Brain. Academic Press, San Diego, pp 81-96

34. Trevarthen C, Kokkinaki T, Fiamenghi G 1999 What infants' imitation communicates. In: Nadel J, Butterworth G (eds) Imitation in Infancy. Cambridge University Press, Cambridge, pp 127-185

35. Nadel J, Guerini C, Peze A, Rivet C 1999 The evolving nature of imitation as a transitory means of communication. In: Nadel J, Butterworth G (eds) Imitation in Infancy. Cambridge University Press, Cambridge, pp 209-234

36. Kugiumutzakis G 1999 Genesis and development of early infant mimesis to facial and vocal models. In: Nadel J, Butterworth G (eds) Imitation in Infancy. Cambridge University Press, Cambridge, pp 36-59

37. Rizzolatti G, Arbib MA 1998 Language within our grasp. Trends Neurosci 21:188 194

38. Dehaene-Lambertz G, Pena M 2001 Electrophysiological evidence for automatic phonetic processing in neonates. Neuroreport 12:3155-3158

39. Naatanen R, Gaillard AW, Mantysalo S 1978 Early selective-attention effect on evoked potential reinterpreted. Acta Psychol (Amst) 42:313-329

40. Huotilainen M, Kujala A, Hotakainen M, Shestakova A, Kushnerenko E, Parkkonen L, Fellman V, Naatanen R 2003 Auditory magnetic responses of healthy newborns. Neuroreport 14:1871-1875

41. Kushnerenko E, Ceponiene R, Balan P, Fellman V, Huotilainen M, Naatanen R 2002 Maturation of the auditory event-related potentials during the first year of life. Neuroreport 13:47-51

42. Arbib M 2005 From monkey-like action recognition to human language: an evolutionary framework for neurolinguistics. Behav Brain Sci (in press)

43. Chen R, Gerloff C, Hallett M, Cohen LG 1997 Involvement of the ipsilateral motor cortex in finger movements of different complexities. Ann Neurol 41:247-254

44. Natale M, Gur RE, Gur RC 1983 Hemispheric asymmetries in processing emotional expressions. Neuropsychologia 21:555-565

45. Adolphs R, Damasio H, Tranel D, Damasio AR 1996 Cortical systems for the recognition of emotion in facial expressions. J Neurosci 16:7678-7687

46. Sackeim HA, Gur RC, Saucy MC 1978 Emotions are expressed more intensely on the left side of the face. Science 202:434-436

47. Fernandez-Carriba S, Loeches A, Morcillo A, Hopkins WD 2002 Functional asymmetry of emotions in primates: new findings in chimpanzees. Brain Res Bull 57:561-564 